\title{
2019 Reviewer Thank You
}

\author{
Bahman Guyuron ${ }^{1}$
}

(C) Springer Science+Business Media, LLC, part of Springer Nature and International Society of Aesthetic Plastic Surgery 2020

I would like to express my deepest gratitude on behalf of the APS editorial staff, Springer Science and Business Media, and International Society of Aesthetic Plastic Surgery (ISAPS), to all the reviewers who have given their valuable time and expertise to this publication, tirelessly and without compensation. Unquestionably, this publication would not enjoy its continued growth and success without the reviewers named in the following list. I look forward to your participation in the revolution of the journal and wish everyone good health and prosperity in the new year.

\section{List of Reviewers}

Ozan Luay Abbas

Lahlali Abdennasser

Yasser Aaboelatta

Ahmed M. Afifi

Frank Agullo

Ovunc Akdemir

Ersin Aksam

Bander Alaithan

Jorge Manuel Albertal

Juan Pablo Aracil Kessler

Luiz Roberto Araujo

Tomer Avraham

Huseyin Ozgur Aytac

Hassan Adel Badran

Semih Baghaki

Yahya Baltu

Venkata Ratnam Bandikatla

Marcus Vinícius Barbosa

Mauro Barone

Alfonso Barrera
Sophie Bartsich

Filipe V. Basile

Mehmet Bayramicli

Devra Becker

Arash Beiraghi-Toosi

Giacomo Bellinvia

Richard Bensimon

Catherine Bergeret-Galley

Nicolas Bertheuil

Ahmet Biçer

Alessandra Bocchiotti

Stefano Bonomi

Alessandro Borgognone

Filippo Boriani

Francisco Borja

Chiara Botti

Giovanni Botti

Robert Bowen

James Franklin Boynton

Mehmet Bozkurt

Bruna Felix Souza Bravo
Francisco G. Bravo

Miguel G. Bravo

Beatriz Brea-García

Ryane Brock

Mitchell H. Brown

Ernesto Maria Buccheri

Marco A. de Camargo Bueno

Antonio Bulla

Sarah Calabrese

Andres Alberto Camayo

Gianluca Campiglio

Marian Cantisano-Zilkha

Salvatore Carlucci

Yanko Castro-Govea

Giuseppe Catanuto

Robert H. Caulfield

Nuri Abdullah Celik

Mehmet Çelik

Patricio Centurion

Candemir Ceran

Mario Ceravolo

Bahman Guyuron

bguyuron@guyuron.com

1 Cleveland, USA 


Nazim Cerkes
Kuo-Hwa Chang
Baoguo Chen
Samuel H.T. Chen
Tien-Hsing Chen
Yu Tsung Chen
Biao Cheng
Cheng-Hung Chiu
Wen-Kuan Chiu
Kang Young Choi
Moon Choi
Sunil Choudhary
Francesco Ciancio
Emanuele Cigna
Yakup Cil
Anı Cinpolat
Maria Cecilia Closs Ono
Yusuf Kenan Coban
Annalisa Cogliandro
Adam J. Cohen
Brian D. Cohen
Steven R. Cohen
Harun Çöloğlu
Andrea Conversi
Eray Copcu
Joao Correia Anacleto
Inês Correia-Sá
Haiyan Cui
Gary R. Culbertson
Andreas Dacho
Francesco D'Andrea
Daniel Melo de Azevedo
Francesco De Francesco
Claudio L. De Lorenzi
Roy De Vita
Nima Dehghani
Erhan Demir
Efterpi Demiri
Rafael Denadai
Pavlov Denyshchuk
Luigi Di Rosa
Isa Dietrich
Andrii Dinets
Jingwen Ding
Denizhan Dizdar
Marek Krzysztof Dobke
Crescenzo D’Onofrio
Muzaffer Durmus
Raeaf El Kollali
Hisham Mahmoud El Minawi
Mohamed Ahmed El-Rouby
Ahmed Elshahat
Onur Erol
Burak Ersoy

Nazim Cerkes

Kuo-Hwa Chang

Baoguo Chen

Samuel H.T. Chen

Tien-Hsing Chen

Yu Tsung Chen

Biao Cheng

Kang Young Choi

Moon Choi

Sunil Choudhary

Francesco Ciancio

Emanuele Cigna

Yakup Cil

Anı Cinpolat

Maria Cecilia Closs Ono

Yusuf Kenan Coban

Annalisa Cogliandro

Adam J. Cohen

Brian D. Cohen

Steven R. Cohen

Eray Copcu

Joao Correia Anacleto

Inês Correia-Sá

Haiyan Cui

Gary R. Culbertson

Andreas Dacho

Francesco D'Andrea

Daniel Melo de Azevedo

Francesco De Francesco

Claudio L. De Lorenzi

Roy De Vita

Nima Dehghani

an Demir

Efterpi Demiri

Rafael Denadai

Luigi Di Rosa

Isa Dietrich

Andrii Dinets

Jingwen Ding

Denizhan Dizdar

Marek Krzysztof Dobke

Crescenzo D'Onofrio

Muzaffer Durmus

Raeaf El Kollali

Hisham Mahmoud El Minawi

Mohamed Ahmed El-Rouby

Onur Erol

Burak Ersoy
Tolga Eryilmaz

Steven Fagien

Dong-Li Fan

Julio Wilson Fernandes

Sergio Fernández-Cossío

Nyer Firdoose

Jack Fisher

Joan Fontdevila

Kenneth Francis

Stanley Paul Frileck

Antonio Fuente Del Campo

Ata Garajei

Julio Luis Garcia

Oihane García Senosiain

Enrique Garcia-Murray

Karin Marcelle Geldenhuys

Lisa Gfrerer

Ashkan Ghavami

Robert Gilman

Salvatore Giordano

João Carlos Sampaio Goes

Mario Goisis

Hiram Osiris Gonzalez

Alejandro Gonzalez-Ojeda

Ruth Maria Graf

Robert Grant

Andreas Gravvanis

Vladimir Grigoryants

Ronald P. Gruber

Katherine Grunzweig

Giuseppe Guarro

Wolfgang Gubisch

Nazım Gümüş

Ethem Guneren

Lifei Guo

Emre Güvercin

Samer Bassilios Habre

Farhad Hafezi

Marc-James Hallam

Dennis Hammond

Ahmed Ali Hassan

Ahmed Sabry Hassen

Daniel A. Hatef

Paul Immanuel Heidekrueger

Benoit Hendrickx

Darryl James Hodgkinson

Alfredo Ernesto Hoyos

Jinjun Huang

Joseph Paul Hunstad

Ran Huo

Dennis J. Hurwitz

Husein Husein-ElAhmed

Bilsev Iince

Alessandro Innocenti
Cristina Isac

Tonguc Isken

Patricio F. Jacovella

Jae Hoon Jeong

Dana Jianu

Gustavo Jimenez Muñoz Ledo

Hong-Ryul Jin

Ung Sik Jin

Janna Joethy

Jamal Jomah

Marija R. Jovic

Valderi Vieira Da Silva Júnior

Abdoljalil Kalantar Hormozi

Gavin Chun-Wui Kang

James Roy Kanjoor

Ercan Karacaoglu

Stefano Andrea Karoschitz

Nolan S. Karp

Anna Kasielska-Trojan

Oguz Kayiran

Yavuz Kececi

Li Kecheng

Noemi Kelemen

Musa Kemal Keleş

Mustafa Keskin

Umar D. Khan

Regina Khater

Hassan Mahmoud Kholosy

Lee Seng Khoo

Kamran Khoobehi

Yong Kyu Kim

Bianca Knoll

Emre Atacan Kocman

Jerzy Roch Kolasinski

Petors Konofaos

Paraskevas Kontoes

Jon Kurkjian

Walter Lampeter

Claude Lassus

Michelle Lee

Myung Chul Lee

Gottfried Lemperle

Zhanqiang Li

Ioannis Liapakis

Chih-Hsun Lin

Samuel J. Lin

Tsai-Ming Lin

Chunjun Liu

Lei Liu

Charles Yuen Yung Loh

Z. Paul Lorenc

Jie Luan

Paulo Henrique Luiz de Freitas

Hsu Ma 


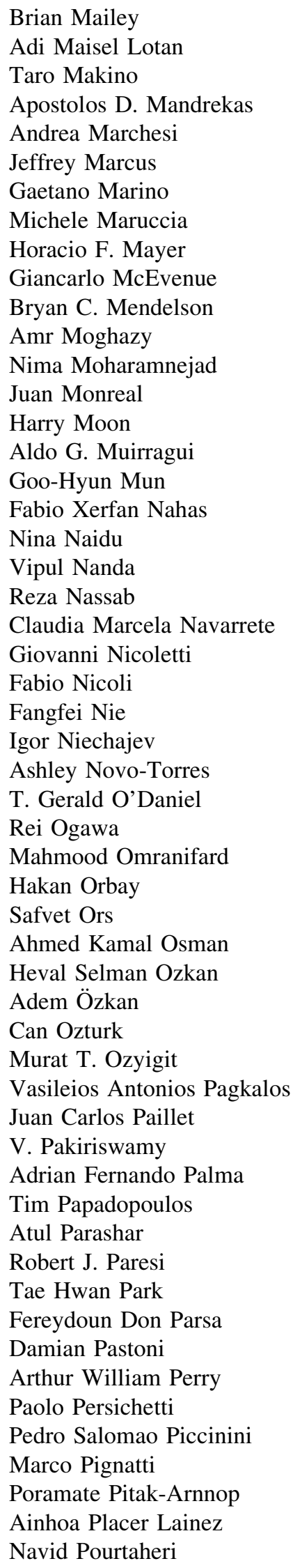

Jason Pozner

Theddeus O.H. Prasetyono

Kirill Pshenisnov

Lee L.Q. Pu

Kimit Rai

Cassio Eduardo Raposo-Amaral

Jose Alexis Reyes

Alfonso Riascos

Ricardo Ribeiro

Dirk Richter

Morris Ritz

Enrico Robotti

Dario Rochira

Tai Suk Roh

Christine Rohde

Andrea Romanzi

Li Rong

Marina Junqueira Rosique

Rodrigo Gouvea Rosique

Percy Rossell-Perry

Min-Hee Ryu

Ahmad Saad

Richard Sadove

Babak Saedi

Osvaldo Ribeiro Saldanha

Jeffrey Salomon

Renato Saltz

Elif Sari

Sezai Sasmaz

Amir Arvin Sazgar

Adam D. Schaffner

Kai-Uwe Schlaudraff

Peter Scott

Cemal Senyuva

Konstantinos Seretis

Jose M. Serra-Renom

Marcos Sforza

Ramesh Kumar Sharma

Jiun-Pyng Shiau

Christo Shipkov

Sachin Shridharani

Raghav Shrotriya

Mattia Siliprandi

Eduardo Nascimento Silva

Pierfranco Simone

Emin Sir

Violeta Skorobac Asanin

Jaime Solis

Graeme Southwick

Luís Ricardo Martinhão Souto

Ozan Sozer

David A. Staffenberg

Ithamar Nogueira Stocchero

Aaron Stone
Howard David Stupak

Gustavo Sturtz

Ross Stutman

In Suck Suh

Man Koon Suh

Daniel Sundfeld Spiga Real

Kashyap Tadisina

Rieka Taghizadeh

Susumu Takayanagi

Sedat Tatar

Goktekin Tenekeci

Oren Tepper

Erdem Tezel

Dinesh Kumar Thekkinkattil

Spero John Theodorou

Claudio Fernando Thomas

Romualdo Suzano Louzeiro Tiago

Jose Alvaro Toribio

Dean Michael Toriumi

Zekeriya Tosun

Ali Totonchi

Mathias Tremp

Lina Triana

Su-Ben Tsao

Umut Tuncel

Carlos Oscar Uebel

Hakan Uzun

David Valauri

Augusto Sette Camara Valente

Berend van der Lei

Ivar van Heijningen

Jan J. van Wingerden

Henry Vasconez

Luis O. Vasconez

Edwin Vidal Vasquez

Guillermo Daniel Vázquez

Ayşegül Verim

Alexis Verpaele

Giovanni Andre Pires Viana

Argentina Vidrascu

Vincenzo Vindigni

Giuseppe Visconti

Fausto Viterbo

Spiros P. Vlachos

Lou Vogel

Krishna S. Vyas

Heidi A. Waldorf

Robert Wallace

Yang Wang

Lara Wetton

Reto Wettstein

Alan David Widgerow

Maria Wiedner

Sebastian Winocour 
Chin-Ho Wong

Guoping Wu

June Wu

Lehao William Wu

Sufan $\mathrm{Wu}$

Allan E. Wulc

Marcelo Wulkan

Zhibo Xiao
Akira Yamada

Zheng Yan

Qingmo Yang

Daniel Vesselov Yankov

Michael J. Yaremchuk

Matthew Sze-Wei Yeo

Guray Yesiladali

Kahraman Berkhan Yılmaz
Yalcin Yontar

Chung-Chih $\mathrm{Yu}$

Dong Yu

$\mathrm{Li} \mathrm{Yu}$

Jonathan Amer Zelken

Lu Zhou

Li Zhuang

Christopher Zoumalan

Publisher's Note Springer Nature remains neutral with regard to jurisdictional claims in published maps and institutional affiliations. 